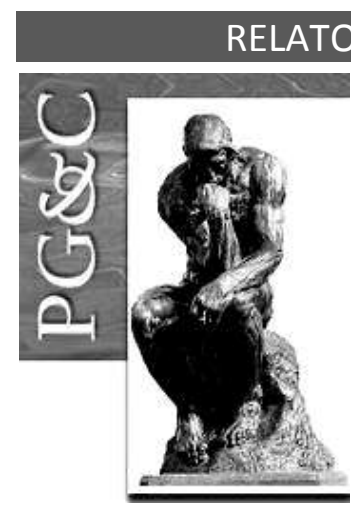

\title{
COMPARTILHAMENTO DE CONHECIMENTO NAS ORGANIZAÇÕES: UM MODELO ANALÍTICO SOB A ÓTICA DA AÇÃO COOPERATIVA
}

\author{
Victor Fraile Sordi \\ Doutorando em Engenharia e Gestão do Conhecimento pela Universidade \\ Federal de Santa Catarina, Brasil. \\ E-mail: victor.sordi@yahoo.com.br \\ Marina Keiko Nakayama \\ Doutora em Administração pela Universidade Federal do Rio Grande do \\ Sul, Brasil. Professora da Universidade Federal de Santa Catarina, Brasil. \\ E-mail: marina@egc.ufsc.br

\section{Erlaine Binotto} \\ Doutora em Agronegócios pela Universidade Federal do Rio Grande do \\ Sul, Brasil. Professora da Universidade Federal da Grande Dourados, Brasil. \\ E-mail: erlainebinotto@ufgd.edu.br
}

\begin{abstract}
Resumo
As organizações necessitam inovar para se sustentar em mercados cada vez mais dinâmicos e competitivos. Compartilhar conhecimento de maneira eficiente se torna essencial nesse contexto, pois é a partir de novos conhecimentos que as inovações fundamentalmente são concebidas. Este estudo tem como objetivo propor um modelo analítico e empírico do compartilhamento de conhecimento nas organizações baseando-se em uma abordagem do compartilhamento de conhecimento como ação cooperativa. Para tanto foi realizada uma revisão crítica dos antecedentes literais sobre o compartilhamento de conhecimento nas organizações. Os resultados sugerem que os principais focos para a análise desse processo são: as características do conhecimento a ser compartilhado, as características do ambiente organizacional, as oportunidades para compartilhar, as barreiras ao compartilhamento, as condições para a existência de cooperação e as motivações para compartilhar. Conclui-se que esse modelo analítico possibilita aos gestores, analistas, pesquisadores e demais interessados, a identificação de problemas relativos ao compartilhamento de conhecimento nas organizações e o planejamento de possíveis soluções, medidas corretivas, ações ou políticas, fundamentadas na realidade de cada organização.
\end{abstract}

Palavras-chave: Cooperação. Gestão do Conhecimento. Compartilhamento de Conhecimento.

\section{KNOWLEDGE SHARING IN ORGANIZATIONS: AN ANALYTICAL MODEL UNDER THE PERSPECTIVE OF COOPERATIVE ACTION}

\begin{abstract}
Organizations need to innovate to stay in increasingly dynamic and competitive markets. Effectively sharing knowledge is essential in this context, once new knowledge fundamentally conceives innovations. This study aims to propose an analytical and empirical model of knowledge sharing in organizations based on a knowledge sharing approach as cooperative action. For this, a critical review of the literal antecedents about the knowledge sharing in organizations was carried out. The results suggest that the main focuses for the analysis of this process are: the characteristics of the knowledge to be shared, the characteristics of the organizational environment, the opportunities to share, the barriers
\end{abstract}

Perspectivas em Gestão \& Conhecimento, João Pessoa, v. 8, n. 1, p. 44-66, jan./abr. 2018. DOI: http://dx.doi.org/10.21714/2236-417X2018v8n1p44.

http://periodicos.ufpb.br/ojs2/index.php/pgc. ISSN: 2236-417X. Publicação sob Licença (cc) EY-NC-ND 
to sharing, the conditions for the existence of cooperation and the motivations to share. This analytical model can enable managers, analysts, researchers and other stakeholders to identify problems related to the sharing of knowledge in organizations and the planning of possible solutions, corrective measures, actions or policies, based on the reality of each organization.

Keywords: Cooperation. Knowledge Management. Knowledge Sharing.

\section{INTRODUÇÃO}

A inovação é alcançada, fundamentalmente, por intermédio da criação de novos conhecimentos (POPADIUK; CHOO, 2006). Para criá-los, as organizações necessitam disponibilizar aos seus funcionários, de forma sistemática e contínua, os conhecimentos tácitos e explícitos adequados (NONAKA, VON KROGH, 2009). Uma das formas de se disponibilizar tais conhecimentos é investindo em processos de compartilhamento de conhecimento entre pessoas e, consequentemente, entre setores, departamentos, elos de uma cadeia produtiva. Essas preocupações fazem parte do escopo da gestão do conhecimento, disciplina a qual engloba uma série de princípios, conceitos, processos, práticas e ferramentas que visam otimizar o uso do conhecimento organizacional para a criação de valor aos stakeholders, sobretudo, criando novos conhecimentos e possibilitando inovações (DOROW; CALLE; RADOS, 2015).

Dentre os processos da gestão do conhecimento, o compartilhamento de conhecimento é encarado como o mais problemático, e tem demonstrado muitas dificuldades em sua implementação (LIN; WU; LU, 2012). Estima-se que as 500 empresas listadas pela revista Fortune perdem ao menos 31,5 bilhões de dólares por ano ao não compartilhar o conhecimento de forma eficaz (ABDUL-CADER; JOHAR, 2015).

Mesmo com as facilidades emergentes das novas tecnologias, no ambiente de trabalho o compartilhamento de conhecimento ainda é um desafio (HONG; SUH; KOO, 2011). Para Davenport e Prusak (2000), isso se deve ao fato de que no ambiente de trabalho não é natural para as pessoas compartilhar conhecimento, visto que os indivíduos tratam o conhecimento possuído como um recurso valioso e significativo.

Diante desse contexto, como a gestão organizacional pode facilitar o compartilhamento de conhecimento entre seus colaboradores? Para tentar responder essa questão o objetivo deste estudo é propor um modelo analítico e empírico do compartilhamento de conhecimento nas organizações baseando-se em uma abordagem do compartilhamento de conhecimento como ação cooperativa.

Partimos do pressuposto de que ao alterar as condições de cooperação entre os indivíduos a gestão organizacional pode estar interferindo positivamente nesses processos (SORDI; BINOTTO; RUVIARO, 2014). Nosso modelo analítico pode possibilitar, aos gestores, analistas, pesquisadores e demais interessados, a identificação de problemas relativos ao compartilhamento de conhecimento nas organizações e o planejamento de possíveis soluções, medidas corretivas, ações ou políticas, fundamentadas na realidade de cada organização.

\section{REFERENCIAL TEÓRICO}

Não há na literatura um consenso quanto ao conceito de compartilhamento de conhecimento. Há autores que o tratam como um processo (SZULANSKI, 2000) outros como ato ou ação (IPE, 2003).

Szulanski (2000) ao tratar da temática, aborda o compartilhamento de conhecimento como um processo, constituído de diferentes estágios, cada estágio com suas dificuldades

Perspectivas em Gestão \& Conhecimento, João Pessoa, v. 8, n. 1, p. 44-66, jan./abr. 2018. 
próprias. Por outro lado, Brachos et al. (2007) e Van den Hooff e Van Weenen (2004), afirmam que $o$ ato de compartilhar o conhecimento implica tanto em doá-lo como em recebê-lo, permitindo ao indivíduo combinar ideias, pontos de vista e informações previamente díspares, tornando possível construir novos conhecimentos a partir dos conhecimentos adquiridos por outros.

Outra peculiaridade do termo na literatura, é sua utilização ambígua que se confunde muitas vezes com "transferência de conhecimentos" ou ainda "troca de conhecimentos" (WANG; NOE, 2010). Antes de se aprofundar na temática, optamos por diferenciar as terminologias e evidenciar a abordagem a ser utilizada.

A transferência de conhecimento para Szulanski (2000) envolve tanto o compartilhamento do conhecimento pela fonte de conhecimento como também a aquisição e aplicação do conhecimento pelo destinatário (receptor). Tal terminologia tipicamente tem sido usada para descrever o movimento, ou fluxo, de conhecimentos entre diferentes unidades, divisões ou organizações, ao invés de indivíduos (SZULANSKI et al.,2004; WANG; NOE, 2010).

Apesar de a terminologia "troca de conhecimentos" ser usada como sinônimo de "compartilhamento de conhecimento" por Brachos et al. (2007), Cabrera, Collins e Salgado (2006) e Van den Hooff e Van Weenen (2004), troca de conhecimentos inclui tanto o compartilhamento de conhecimento - a fonte repassando o conhecimento ao destinatário como também a busca por conhecimento, ou seja, o indivíduo ativamente buscando os conhecimentos de seus pares.

Já o "compartilhamento de conhecimento" é o ato de tornar o conhecimento disponível para os outros, entre indivíduos esse processo permite uma pessoa transformar conhecimento para que seja entendido, absorvido e útil para outros atores (IPE, 2003).

Em complemento, Bartol e Srivastava (2002) definem compartilhamento de conhecimento como sendo o compartilhamento de informações, ideias, sugestões e experiências relevantes aos indivíduos de uma determinada organização. Ou seja, o compartilhamento de conhecimento no sentido mais restrito do termo, refere-se somente a ação em que um indivíduo disponibiliza seus conhecimentos para seus pares, desta forma, a busca pelo conhecimento feito por um indivíduo ou a transferência coletiva de conhecimentos entre organizações não são contempladas pelo conceito (WANG; NOE, 2010).

Este estudo pretende tratar do compartilhamento de conhecimento, tanto na dimensão individual como na dimensão organizacional. Dessa forma, a abordagem utilizada tratará a terminologia "compartilhamento de conhecimento" como o ato de disponibilizar o conhecimento para outros indivíduos, assim como a busca por esse conhecimento e o seu fluxo entre setores, departamentos, organizações, elos de uma cadeia produtiva.

Nesse sentido, Cummings (2004) e Pulakos, Dorsey e Borman (2003) referem-se ao compartilhamento de conhecimento como fornecimento de know-how e informações quanto a tarefas para ajudar seus pares e colaborar na resolução de problemas, desenvolvendo novas ideias ou implementando novas políticas ou procedimentos. Podemos perceber por esta definição conceitual e por outras citadas neste tópico (como exemplo: IPE, 2003), que o compartilhamento de conhecimento pode ser idealizado como um ato colaborativo. As definições conceituais apresentadas se aproximam da ideia de que o processo é ou deveria ser amistoso, em que as fontes e destinatários desse ato de compartilhar, se ajudariam e se beneficiariam com os resultados.

Entretanto, como expressa Davenport e Prusak (2000), o processo de compartilhar conhecimento no contexto organizacional não é tão natural como demonstrado nas interpretações amistosas dos conceitos apresentados. À medida que os indivíduos vislumbram um valor significativo nos conhecimentos possuídos, os atos/processos de compartilhamento desses "valiosos" conhecimentos com seus pares, podem ser prejudicados por "barreiras" ao compartilhamento de conhecimento (HONG; SUH; KOO, 2011).

Perspectivas em Gestão \& Conhecimento, João Pessoa, v. 8, n. 1, p. 44-66, jan./abr. 2018. 
Por esse motivo o compartilhamento de conhecimento é alvo de diversas pesquisas tanto qualitativas, como quantitativas, em várias áreas do conhecimento como: contabilidade; astronomia; biologia; negócios; comunicação; criatividade; economia; educação; engenharia; ergonomia; saúde; direito; biblioteconomia; gestão; filosofia; psicologia e trabalho social (WITHERSPOON, 2013).

Mesmo quando discutido na perspectiva da gestão do conhecimento organizacional, como é o caso neste estudo, são diversas as abordagens e teorias utilizadas na literatura. Dentre elas podemos destacar:

(1) Teoria da Ação Racional (BOCK et al., 2005; CABRERA; CABRERA, 2005; RYU; HO; HAN, 2003) sugere que um determinado comportamento, por exemplo, o de compartilhar conhecimento, é fruto de uma intenção em realizá-lo e essa intenção é influenciada pela atitude do indivíduo, sendo essa determinada por crenças e normas subjetivas em relação ao comportamento. Em outras palavras, a intenção de se envolver em um comportamento de compartilhamento de conhecimento é determinada por avaliações e crenças sobre os resultados desse comportamento (atitude), bem como por percepções de normas sociais (pressão por comportamentos esperados) no contexto onde o indivíduo está inserido;

(2) Teoria do Comportamento Planejado (TCP) (HSU; LIN, 2008; LIN, 2007; RYU; HO; HAN, 2003) se apropria das contribuições da teoria da ação racional e as complementa com os aspectos relativos ao controle comportamental percebido pelo indivíduo. Essa teoria indica fatores motivacionais que podem influenciar o comportamento, seguido de quanto esforço os indivíduos estão dispostos a investir para realizar o comportamento e, finalmente, até que ponto iriam para concretizar tal comportamento. Em última análise, segundo a TCP, se o indivíduo tem a intenção de compartilhar conhecimento com seus pares, ele provavelmente irá compartilhar. E que essa intenção é influenciada pelo controle comportamental percebido por esse indivíduo, além de sua atitude perante as crenças e normas subjetivas percebidas.

(3) Teoria dos Dilemas Sociais (CABRERA; CABRERA, 2005; CASIMIR; LEE; LOON, 2012; ZÁRRAGA; BONACHE, 2003) sugere que um indivíduo irá compartilhar conhecimento com seus pares, somente se conseguir ilações de que esse comportamento Ihe trará mais benefícios do que custos. Em outras palavras, o indivíduo necessita perceber vantagens em compartilhar conhecimento e contribuir com seus pares, para que no dilema social de compartilhar ou não compartilhar, opte por compartilhar;

(4) Teoria das Trocas Sociais (CABRERA; CABRERA, 2005; HSU; LIN, 2008; LIAO, 2008) afirma que os indivíduos regulam suas interações com outros indivíduos com base em uma análise de "auto interesse" dos custos e benefícios relacionados as interações. Desse modo, conforme a teoria, o compartilhamento de conhecimento será afetado positivamente quando um indivíduo confia que esse comportamento será retribuído com algum benefício no futuro. Ou seja, quando o indivíduo percebe que as trocas sociais (interações) serão vantajosas;

(5) Teoria do Capital Social (CABRERA; CABRERA, 2005; CHIU; HSU; WANG, 2006; HSU; CHANG, 2014) capital social - entendido como o conjunto de normas de reciprocidade, informação e confiança presentes nas redes sociais informais desenvolvidas pelos indivíduos em sua vida cotidiana - é um grande facilitador dos processos de compartilhamento. Nessa perspectiva, o capital social existente em determinado contexto facilita as interações entre os envolvidos que são necessárias para a ação coletiva bem-sucedida. Nesse caso o compartilhamento de conhecimento. Em outras palavras, laços sociais, linguagem 
compartilhada, oportunidade de compartilhar, tempo de contato e estrutura das interações podem influenciar diretamente no compartilhamento de conhecimento entre as pessoas; e

(6) Abordagem motivação-oportunidade-capacidade (ARGOTE; MCEVILY; REAGANS, 2003; CHEN; CHANG; TSENG, 2012; SIEMSEN; ROTH; BALASUBRAMANIAN, 2008) O desempenho do indivíduo no trabalho pode ser explicado por sua motivação, pelas oportunidades a ele dadas e pela sua capacidade. No contexto do compartilhamento de conhecimento a motivação capta a disposição do indivíduo em compartilhar, a oportunidade representa os mecanismos ambientais ou contextuais que permitem o compartilhamento e a capacidade representa as competências e habilidades do indivíduo em compartilhar, ou ainda a base de conhecimento relacionada às suas capacidades de compartilhar conhecimentos.

Sordi, Binotto e Ruviaro (2014), ao relacionarem a cooperação ao compartilhamento de conhecimento, apresentam evidências de que num contexto organizacional competitivo a cooperação depende do compartilhamento de conhecimentos para existir e o próprio compartilhamento de conhecimento necessita da cooperação entre os agentes para acontecer. Nessa perspectiva, o compartilhamento de conhecimento necessita de determinadas condições para acontecer. Uma delas é a possibilidade de cooperação entre os agentes envolvidos (fontes e destinatários de conhecimento).

Ao relacionarmos essa perspectiva com as principais teorias utilizadas nas publicações consultadas, percebemos que ela se apropria de contribuições da Teoria da Ação Racional e do Comportamento Planejado, pois indica que a cooperação ocorre quando os indivíduos possuem a intenção em cooperar pela sua percepção das normas, crenças, pressões sociais e comportamentais existentes. Assim como, assimila contribuições da Teoria das Trocas Sociais e Teoria dos Dilemas Sociais, visto que sugere que o indivíduo coopera quando percebe vantagens e benefícios maiores do que custos e desvantagens.

Sob essa ótica, propomos, neste estudo, tratar o compartilhamento de conhecimento como uma ação cooperativa.

\subsection{O compartilhamento de conhecimento como ação cooperativa}

Uma ação social é baseada nas ações e reações de outros indivíduos e pode ser modificada a partir desses eventos (WEBER, 1978). Dessa forma trata-se da conduta humana a que um indivíduo atribui significados subjetivos. Compreender esses significados é um dos focos da sociologia, assim como os processos sociais, dentre os quais, a cooperação entre as pessoas (DURKHEIM, 2001).

A cooperação nesse sentido trata-se de uma ação social e como qualquer ação social em seu sentido mais amplo, pode ser compreendida como uma transação, ou seja, uma interação entre dois ou mais atores, formal ou informal, mercantil ou não mercantil (RICHTER, 2015). Nessa perspectiva a cooperação é construída por interesses mútuos entre os indivíduos que optam por cooperar vislumbrando maiores ganhos numa interação cooperativa do que em uma interação sem cooperação (GRASSI, 2006; VILPOUX, 2014).

O compartilhamento de conhecimento nesse contexto, embora não seja uma simples transação com troca de ativos entre as partes, é uma interação entre dois ou mais indivíduos em que o conhecimento para ser compartilhado necessita de condições adequadas já que se trata de um recurso valioso e significativo para as pessoas (DAVENPORT; PRUSAK, 2000).

Dessa maneira, assim como na cooperação, o ato de compartilhar está ligado aos interesses mútuos (IPE, 2003) dos atores envolvidos e as suas respectivas percepções quanto aos benefícios e custos envolvidos nesse processo (LIN; WU; LU, 2012).

Perspectivas em Gestão \& Conhecimento, João Pessoa, v. 8, n. 1, p. 44-66, jan./abr. 2018. 
Ao compartilhar seu conhecimento o indivíduo está cooperando com o destinatário do conhecimento na medida em que os benefícios vislumbrados desse ato sejam maiores que os custos (NOWAK, 2006; SORDI; BINOTTO; RUVIARO, 2014). Baseando-se na confiança (IPE, 2003), na reciprocidade (NOWAK, 2006), dentre outros aspectos correlatos, o indivíduo fonte do conhecimento a ser compartilhado decide se coopera ou não com o destinatário do ato e consequentemente compartilha ou não seu conhecimento com o outro (SORDI; BINOTTO; RUVIARO, 2014).

Assim sendo, ao compartilhar conhecimento o indivíduo considerado como a fonte está disponibilizando um ativo intangível (SZULANSKI, 2000) para um ou mais indivíduos destinatários desse conhecimento, logo está desempenhando uma interação social que sendo uma espécie de transação (WILLIAMSON, 1996) está vulnerável a custos, riscos e incertezas (RICHTER, 2015) mesmo que mínimos e irrelevantes.

Assim, ao compartilhar um conhecimento que tenha grande utilidade em alguma atividade específica dentro das organizações, a fonte do conhecimento a ser partilhado está cooperando com o destinatário na medida em que se não houvesse algum benefício nesse ato, a fonte não estaria propensa a efetivá-lo (LIN; WU; LU, 2012; SALIM et al.,2011; SORDI; BINOTTO; RUVIARO, 2014)

Tais benefícios percebidos podem ser de naturezas variadas como na reciprocidade do ato em uma oportunidade futura (SORDI; BINOTTO; RUVIARO, 2014), um incentivo monetário em troca do conhecimento partilhado ou ainda outro conhecimento a ele útil dentro do mesmo contexto. Assim como no caso dos benefícios percebidos, os custos podem ter múltiplas naturezas como o receio em expor um ativo intangível valioso (DAVENPORT; PRUSAK, 2000), o tempo gasto no compartilhamento (ROSEN; FURST; BLACKBURN, 2007; SANTOS; SOARES; CARVALHO, 2012) ou a falta de reciprocidade (oportunismo) do ato por parte dos destinatários do conhecimento.

Por outro lado, os destinatários também podem perceber custos e benefícios em recepcionar o conhecimento partilhado pelas fontes. Pode-se categorizar como custos envolvidos na recepção: o tempo utilizado (ROSEN; FURST; BLACKBURN, 2007), o risco de inutilidade do conhecimento (ARDICHVILI et al., 2006), a dificuldade em absorvê-lo e interpretá-lo (HSU; CHANG, 2014), a distância entre fonte e destinatário ou a falta de confiança no uso depois de aprendido (CABRERA; COLLINS; SALGADO, 2006). Em contrapartida, benefícios como ganhos advindos do usufruto dos novos conhecimentos, status almejado com determinado conhecimento ou ainda possibilidades de ganhos monetários com a aquisição de novos conhecimentos podem ser determinantes para a efetiva partilha entre esses indivíduos (WANG; NOE, 2010).

Dessa forma, mesmo com a utilização de repositórios de conhecimento, tecnologias de informação e outras oportunidades facilitadas de compartilhamento, se não houver cooperação entre as partes o compartilhamento de conhecimentos essenciais para as organizações provavelmente não irá acontecer com efetividade (HENDRIKS, 1999; SORDI; BINOTTO; RUVIARO, 2014).

Nesse sentido, a motivação de compartilhar conhecimento estará relacionada aos custos e benefícios percebidos pelos envolvidos e, consequentemente, a disponibilidade de cooperação entre eles através de interesses comuns mesmo que por objetivos distintos. Sob essa perspectiva, as barreiras ao compartilhamento são custos do ato de cooperação, enquanto os fatores facilitadores sejam organizacionais, individuais ou tecnológicos são os pontos relacionados à minimização desses custos e a maximização dos benefícios percebidos.

Perspectivas em Gestão \& Conhecimento, João Pessoa, v. 8, n. 1, p. 44-66, jan./abr. 2018. 


\section{METODOLOGIA}

A metodologia utilizada foi uma revisão narrativa dos antecedentes literais sobre o compartilhamento de conhecimento nas organizações. Que consistiu em revisitar publicações sobre a temática, sob a ótica da ação cooperativa. Desse modo, este estudo exploratório, utilizou tanto publicações de natureza teórica, como publicações de natureza empírica.

Uma revisão narrativa é uma revisão ampla, extensiva e qualitativa da literatura, apropriada para descrever e discutir o estado da arte de uma determinada temática. $\mathrm{O}$ seu objetivo é basicamente, fornecer uma análise crítica e pessoal dos autores sobre a literatura disponível (ROTHER, 2007). As revisões narrativas não informam as fontes de informação utilizadas, a metodologia para busca das referências, nem os critérios utilizados na avaliação e seleção dos trabalhos (BOTELHO; CUNHA; MACEDO, 2011). Essas escolhas são pessoais e subjetivas de acordo com o senso crítico dos autores.

Ao analisar criticamente as evidências empíricas e as discussões teóricas disponíveis na literatura, percebemos conforme descrito na subseção 4.1, algumas tendências recorrentes nos estudos sobre a temática. Essas tendências revelam uma lógica dominante nessas publicações. Baseando-se nessa lógica dominante, construímos um modelo (Figura 1) para análises empíricas, sob a abordagem da ação cooperativa.

Esse modelo analítico inova ao incorporar a abordagem da ação cooperativa à lógica dominante no estado da arte sobre o compartilhamento de conhecimento nas organizações. $E$ parte da premissa de que a gestão pode interferir positivamente nos processos de compartilhamento de conhecimento ao alterar as condições de cooperação existentes entre os indivíduos.

Os gestores, analistas, pesquisadores e demais interessados podem utilizar o modelo proposto para identificar problemas relativos ao compartilhamento de conhecimento nas organizações e planejar possíveis soluções, medidas corretivas, ações ou políticas, fundamentadas na realidade de cada organização.

\section{RESULTADOS}

Nesta seção o modelo analítico construído a partir da revisão proposta e baseado na abordagem do compartilhamento de conhecimento como ação cooperativa é apresentado.

\subsection{Um modelo analítico baseado na lógica dominante nos antecedentes literais}

Ao revisitarmos a literatura sob o ponto de vista da ação cooperativa percebemos algumas tendências como o foco em fatores que podem influenciar o compartilhamento de conhecimento positivamente, no caso dos facilitadores (AL SAIFI et al., 2016; CAVALIERE; LOMBARDI; GIUSTINIANO, 2015; KIM; NEWBY-BENNETT; SONG, 2012; MA et al., 2014), e negativamente, das barreiras (RIEGE, 2005; SORDI et al., 2017; WANG; NOE, 2010; WITHERSPOON et al., 2013).

Outra tendência percebida foi a classificação recorrente desses fatores em fatores individuais, organizacionais e tecnológicos (LIN, 2007; RIEGE, 2005; SENTHIL VELMURUGAN; KOGILAH; DEVINAGA, 2010; YUSOF et al., 2012).

Ao aprofundarmos na análise destas tendências percebemos que há uma lógica dominante nos estudos sobre compartilhamento de conhecimento nas organizações. Esta lógica evidencia ao menos quatro pontos de convergência na literatura:

I. Há barreiras tanto para quem disponibiliza o conhecimento como para quem busca ou recebe conhecimentos (SORDI et al., 2017). No contexto

Perspectivas em Gestão \& Conhecimento, João Pessoa, v. 8, n. 1, p. 44-66, jan./abr. 2018. 
organizacional as pessoas podem deixar de compartilhar conhecimento por uma série de fatores como falta de tempo disponível (BLOICE; BURNETT, 2016), falta de confiança (RIEGE, 2005), auto eficácia do conhecimento (CAVALIERE; LOMBARDI; GIUSTINIANO, 2015), baixa capacidade de absorção (HSU; CHANG, 2014), layout inadequado (YUSOF, 2012), cultura organizacional que favorece a retenção ou centralização de conhecimento (WITHERSPOON, 2013), dentre outras barreiras que atrapalham tanto fontes como destinatários (RUTTEN et al., 2016; SORDI et al., 2017; ZHANG; JIANG, 2015).

II. Não existem práticas de (KS) infalíveis ou que funcionem em todos os contextos ou ambientes organizacionais. A eficácia dos facilitadores utilizados pela gestão das organizações depende fundamentalmente das características e peculiaridades de cada organização (LILLEOERE; HOLME HANSEN, 2011; RIEGE, 2005). Um sistema de recompensas, por exemplo, pode não funcionar em um determinado contexto, cultura ou tipo de trabalho (BOCK et al., 2005; STENIUS et al., 2016) e funcionar bem em outro (KIM; LEE, 2006, YUSOF, 2012).

III. As características do conhecimento a ser compartilhado podem determinar o sucesso ou o insucesso de um facilitador. As dificuldades da organização em promover o compartilhamento de conhecimento vão depender também do tipo de conhecimento a ser compartilhado (IPE, 2003, RIEGE, 2005), da utilidade do conhecimento em um determinado contexto (HALL, 2001), do valor do conhecimento (IPE, 2003) em determinada situação, contexto ou condição e de sua qualidade (KYOON YOO, 2011).

IV. As barreiras podem funcionar como custos percebidos (CASIMIR; LEE; LOON, 2012). E os facilitadores tentam reduzir esses custos (LILLEOERE; HOLME HANSEN, 2011), buscando oferecer condições vantajosas para quem compartilha conhecimento na organização. Interferindo na motivação dos indivíduos a compartilhar (STENIUS et al., 2016).

V. Oferecer oportunidades de compartilhar conhecimentos com forma, quantidade e qualidade adequadas são fundamentais para a efetivação desses processos e a aderência ao comportamento de compartilhar (IPE, 2003; LAWSON et al., 2009; NONAKA; KONNO, 1998). Não basta estar propenso a compartilhar se as oportunidades forem escassas ou mal construídas.

Esses pontos de convergência foram incorporados no modelo de análise (Figura 1), que apresenta seis elementos principais a serem analisados quanto ao compartilhamento de conhecimento nas organizações: (1) Características do conhecimento a ser compartilhado, (2) Características do Ambiente Organizacional, (3) Oportunidades para compartilhar, (4) Barreiras ao compartilhamento, (5) Condições para a existência de cooperação e (6) Motivações para compartilhar. 
Figura 1 - Modelo de Análise do Compartilhamento de Conhecimento como Ação Cooperativa

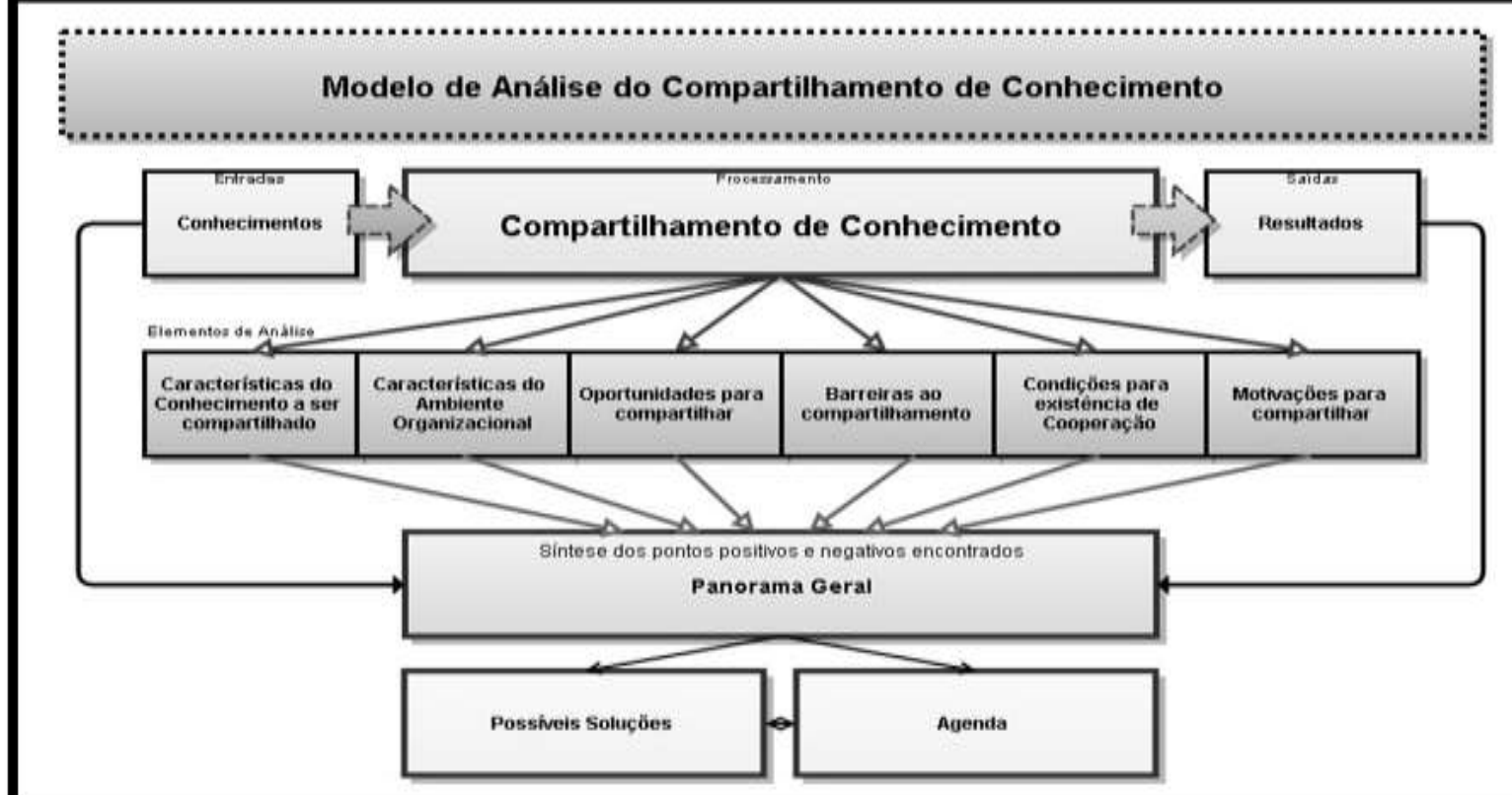

Fonte: Elaborado pelos autores (2018)

Os elementos: (a) "características do conhecimento a ser compartilhado", (b) "características do ambiente organizacional", (c) "oportunidades para compartilhar", (d) barreiras ao compartilhamento" e (e) "motivações para compartilhar", foram incorporados a partir dos pontos de convergência encontrados na literatura e das premissas (Subseção 2.1) da abordagem do compartilhamento de conhecimento como ação cooperativa.

Elemento (a) ponto de convergência III, Elemento (b) ponto de convergência II, Elemento (c) ponto de convergência $V$, Elemento (d) ponto de convergência I, Elemento (e) ponto de convergência IV. Já o elemento "Condições para existência de cooperação" foi incorporado ao modelo como premissa fundamental da abordagem da ação cooperativa.

O conjunto desses elementos faz parte de um sistema onde as "entradas" são os conhecimentos, o "processamento" ou "transformação" é o próprio compartilhamento de conhecimento e as "saídas" são os resultados, tanto quanto ao processo de compartilhar conhecimento como ao desempenho do processo produtivo da organização (VON BERTALANFFY, 2008; YUSOF et al., 2012).

Dessa maneira, ao analisarmos esses elementos dentro de um determinado contexto organizacional, o modelo pode ajudar a compreender um panorama da organização estudada em relação as práticas, condições, iniciativas, pontos fortes e fraquezas quanto ao compartilhamento de conhecimento.

A síntese dos pontos positivos e negativos encontrados apresentam um "panorama geral" da organização quanto ao compartilhamento de conhecimento. Ou seja, após a reunião das informações acerca dos elementos do modelo, esse conjunto analítico de informações constituirá um cenário (panorama). Com esse panorama caracterizado, o analista poderá planejar "possíveis soluções" que serão as intervenções planejadas com base no panorama. E implantar uma "agenda", um cronograma com medidas, ações e políticas que visem interferir positivamente nesses processos.

Essas informações têm em vista a sistematização do processo de compartilhamento de conhecimento, analisando-o como um sistema "retroalimentável". Os seis elementos de

Perspectivas em Gestão \& Conhecimento, João Pessoa, v. 8, n. 1, p. 44-66, jan./abr. 2018. 
análise discutidos a partir dos aportes teóricos adotados podem ser analisados em conjunto, sem, contudo, necessitarem de uma hierarquização padronizada entre eles.

O intuito foi de aproveitar a sobreposição entre as análises de cada elemento. Como por exemplo, as possíveis relações entre as características do ambiente organizacional e as motivações identificadas para compartilhar, ou entre as oportunidades para compartilhar e as condições existentes para cooperação identificadas. No Quadro 1 apresentamos uma síntese dos elementos do modelo.

\begin{tabular}{|c|c|}
\hline \multicolumn{2}{|c|}{ Quadro 1 - Elementos do Modelo } \\
\hline ELEMENTOS & SÍNTESE \\
\hline Entradas & Conhecimentos a serem compartilhados. \\
\hline Saídas & Resultados relacionados ao compartilhamento. \\
\hline Processamento & O próprio processo de compartilhamento. \\
\hline $\begin{array}{l}\text { Características do Conhecimento a ser } \\
\text { compartilhado }\end{array}$ & Propriedades do conhecimento a ser compartilhado. \\
\hline Características do Ambiente Organizacional & Contexto em que o conhecimento será compartilhado. \\
\hline Oportunidades para compartilhar & $\begin{array}{l}\text { Possibilidades de interações com compartilhamento } \\
\text { de conhecimento disponibilizadas. }\end{array}$ \\
\hline Barreiras ao compartilhamento & $\begin{array}{c}\text { Empecilhos ao processo de compartilhamento de } \\
\text { conhecimento, tanto para fontes como para } \\
\text { destinatários. }\end{array}$ \\
\hline Condições para a existência de Cooperação & $\begin{array}{l}\text { Estrutura condicional que possibilita ou prejudica } \\
\text { interações cooperativas com compartilhamento de } \\
\text { conhecimentos. }\end{array}$ \\
\hline Motivações para compartilhar & $\begin{array}{l}\text { Percepções relacionadas ao processo de } \\
\text { compartilhamento de conhecimento. }\end{array}$ \\
\hline Panorama Geral & Cenário com a síntese da análise dos elementos. \\
\hline Possíveis Soluções & Intervenções planejadas com base no panorama geral. \\
\hline Agenda & Cronograma com os passos a serem executados. \\
\hline
\end{tabular}

Fonte: Elaborado pelos autores (2018)

Os analistas (pesquisadores, gestores, consultores e demais interessados) podem optar por formas distintas de captura dessas informações, seja por entrevistas, observações, análise de documentos, pesquisa participante, dentre outros métodos. Nas seções a seguir detalharemos cada um dos seis elementos de análise que compõem o modelo, no sentido de descrever um panorama para futuras intervenções positivas.

\subsection{Características do conhecimento a ser compartilhado}

Percebemos nos antecedentes consultados que um aspecto essencial para analisarmos o processo de compartilhamento de conhecimento é sabermos as características do

Perspectivas em Gestão \& Conhecimento, João Pessoa, v. 8, n. 1, p. 44-66, jan./abr. 2018. 
conhecimento a ser compartilhado (IPE, 2003). De quais conhecimentos estamos falando? Qual o valor desse conhecimento? Tratam-se dos conhecimentos essenciais para o processo produtivo da organização? De qual tipo de conhecimento se trata? Como podemos compartilhá-lo? A qualidade desses conhecimentos é adequada aos objetivos da organização? Onde está esse conhecimento?

$\mathrm{Na}$ perspectiva adotada neste estudo, dependendo das características do conhecimento a ser compartilhado, os custos envolvidos nas interações serão maiores ou menores que os benefícios, viabilizando ou não a escolha por uma interação cooperativa. Ou seja, a opção pelo compartilhamento do conhecimento por parte das fontes e destinatários envolvidos no processo podem variar conforme as características do conhecimento.

A tipologia do conhecimento, por exemplo, pode influenciar todo o processo de compartilhamento (IPE, 2003). O conhecimento a ser partilhado pode ser tácito ou explícito (NONAKA; KONNO, 1998). Os conhecimentos explícitos, como os descritos em manuais, livros com técnicas e procedimentos compilados em programas de computador, são mais facilmente compartilhados exigindo menos esforço para a organização armazenar e compartilhar com seus colaboradores.

Entretanto, os conhecimentos tácitos incorporados às pessoas são de difícil verbalização, necessitando de maior esforço organizacional, podendo ser compartilhados facea-face por intermédio da observação por exemplo.

A utilidade do conhecimento é outra característica importante no compartilhamento de conhecimento (HALL, 2001). As pessoas envolvidas nos processos devem ter a devida noção da utilidade do conhecimento (auto eficácia) a ser compartilhado e a organização pode analisar se os conhecimentos a serem partilhados são realmente úteis aos envolvidos.

Relacionado a utilidade do conhecimento, o valor do conhecimento também é outra característica influente nos processos de compartilhamento (IPE, 2003). Conhecimentos visualizados como de alto valor em determinados contextos são mais difíceis de serem compartilhados do que conhecimentos menos valorizados.

Assim como a utilidade e o valor, a qualidade dos conhecimentos (CABRERA; COLLINS; SALGADO, 2006) a serem compartilhados pode também impactar na efetivação do processo. Se a qualidade do conhecimento disponibilizado é baixa, os destinatários poderão passar a não utilizar tais conhecimentos e "boicotar" os processos de compartilhamento. Assim como, os resultados do processo produtivo podem ser abaixo do esperado, justamente pela fragilidade dos conhecimentos compartilhados na organização.

Sejam conhecimentos tácitos ou explícitos, valiosos, úteis ou de satisfatória qualidade, as fontes de conhecimento e a noção de onde podem ser localizadas, são essenciais para o processo de compartilhamento (BARBOSA et al., 2012).

Em síntese, esse elemento de análise visa oferecer ao analista a possibilidade de caracterizar os conhecimentos a serem compartilhados e avaliar se a organização oferece as condições necessárias para que os envolvidos compartilhem tais conhecimentos. Além das características do conhecimento a ser compartilhado, as características do ambiente organizacional também podem ser fundamentais no condicionamento da cooperação entre os envolvidos e, por consequência, no compartilhamento de conhecimento entre eles.

\subsection{Características do Ambiente Organizacional}

Estudos anteriores sugerem que a cultura (KIM; LEE, 2006), o clima (BOCK et al., 2005), as normas subjetivas (WITHERSPOON et al., 2013), os incentivos (HE; WEI, 2009), o apoio da gestão (LIN, 2007), a estrutura organizacional (RIEGE, 2005), o layout (YUSOF et al., 2012), dentre outras características do ambiente organizacional, impactam direta ou indiretamente nos processos de compartilhamento de conhecimento.

Perspectivas em Gestão \& Conhecimento, João Pessoa, v. 8, n. 1, p. 44-66, jan./abr. 2018. 
Tais aspectos, na perspectiva do compartilhamento de conhecimento como ação cooperativa, fornecem o contexto, o ambiente e as condições necessárias para que as pessoas envolvidas optem por interações cooperativas com seus pares e compartilhem seus conhecimentos. O ambiente organizacional está favorecendo ou prejudicando o compartilhamento de conhecimento?

A cultura organizacional, ou seja, o conjunto de hábitos e crenças estabelecidos através das normas, valores, atitudes e expectativas compartilhadas pelos colaboradores, pode gerar um sistema de significados compartilhados que podem favorecer ou prejudicar a cooperação entre os envolvidos (KIM; LEE, 2006). E, consequentemente, facilitar ou dificultar o compartilhamento de conhecimento.

Uma cultura mais colaborativa, mais aberta aos erros, e caracterizada pelo feedback contínuo, provavelmente irá facilitar as interações cooperativas e o compartilhamento de conhecimento (SANTOS; SOARES; CARVALHO, 2012). Enquanto uma cultura mais rígida e menos suscetível a erros, pode levar os colaboradores a ter receio em arriscar e inovar inviabilizando o compartilhamento de novos conhecimentos. Esse clima competitivo pode causar dificuldades para a cooperação (SORDI; BINOTTO; RUVIARO, 2014) entre diferentes setores, entre filiais, departamentos, dificultando o compartilhamento de conhecimentos (RIEGE, 2005).

Outro aspecto tratado na literatura são as normas subjetivas que se referem à pressão social exercida sobre o indivíduo a fim de que ele se comporte ou não de determinada forma (BOCK et al., 2005). A institucionalização de uma cultura de compartilhamento, por exemplo, pode criar uma pressão normativa social para que os colaboradores compartilhem seus conhecimentos. O que provavelmente facilitará o compartilhamento de conhecimentos entre os envolvidos.

Essa mesma linha de raciocínio pode ser aplicada aos demais aspectos que caracterizam o ambiente de uma organização, como quando uma empresa busca incentivar através de premiações novas ideias para a redução do desperdício. Ou busca incentivar o compartilhamento dessas novas ideias em determinados setores (HE; WEI, 2009). Esses incentivos provavelmente estarão facilitando o compartilhamento de conhecimento criando um ambiente propício por intermédio do apoio da gestão (LIN, 2007).

Em síntese, esse elemento de análise pode oferecer ao analista um panorama da organização focalizada quanto ao contexto atual do ambiente de trabalho. Possibilitando assim, a visualização de quais características do ambiente estão prejudicando ou beneficiando os processos de compartilhamento de conhecimento. $\mathrm{E}$, consequentemente, onde a gestão da organização pode interferir positivamente.

Diretamente relacionadas às características do ambiente organizacional, as oportunidades de compartilhar conhecimento podem ser fomentadas e cultivadas para que os processos de compartilhamento de conhecimento e a cooperação entre os agentes sejam facilitados.

\subsection{Oportunidades para compartilhar}

As oportunidades de compartilhamento, sua forma, qualidade e quantidade podem definir se os envolvidos nos processos de compartilhamento de conhecimento irão compartilhar ou não com seus pares (IPE, 2003). Quando e como o conhecimento está sendo compartilhado? A organização oferece oportunidades para o compartilhamento? A organização utiliza canais adequados para o tipo de conhecimento a ser compartilhado? As pessoas envolvidas nesses processos possuem tempo e recursos adequados para compartilharem conhecimento?

Perspectivas em Gestão \& Conhecimento, João Pessoa, v. 8, n. 1, p. 44-66, jan./abr. 2018. 
Na perspectiva do compartilhamento de conhecimento como ação cooperativa, essas oportunidades além de funcionarem como custos (quando problemáticas) e benefícios (quando bem construídas), também podem criar as condições para a cooperação entre os indivíduos fomentando a confiança (IPE, 2003), a reciprocidade (NOWAK, 2006) e as relações de dependência (VILPOUX, 2014), por intermédio de interações frequentes e duradouras (AXELROD, 1997).

Essas oportunidades podem ser via mecanismos formais como reuniões, sessões de brainstorming, avaliação de projetos anteriores, treinamentos, workshops, intranet e portais corporativos, ou via mecanismos informais como confraternizações, cafés, happy hour, comunidades de prática, fóruns, redes sociais e plataformas wiki (LAWSON et al., 2009). Seja através de algum tipo de canal de relacionamento (IPE, 2003) ou com a utilização de plataformas de interação (NONAKA; KONNO, 1998), a organização pode analisar qual a melhor forma para compartilhar os conhecimentos que necessita, oferecendo sistematicamente oportunidades e recursos adequados para efetivação dos processos de compartilhamento.

Conforme os antecedentes consultados neste estudo, ao optar por canais de relacionamento e plataformas virtuais, as organizações devem atentar para a disponibilidade desses sistemas (CABRERA; COLLINS; SALGADO, 2006). A tecnologia de informação e comunicação (sistemas, repositórios, intranet) deve estar disponível sempre que necessário, oferecendo acesso facilitado e rápido as informações e conhecimentos (SENTHIL VELMURUGAN; KOGILAH; DEVINAGA, 2010).

Além da disponibilidade, a literatura aponta a adequação dos recursos tecnológicos (SENTHIL VELMURUGAN; KOGILAH; DEVINAGA, 2010). Sejam equipamentos, softwares ou servidores, esses devem ser adequados aos objetivos e demandas dos colaboradores (LIN et al., 2009). Caso contrário, se tornam barreiras ao compartilhamento de conhecimentos e ao invés de promover maior número de interações entre os funcionários, podem isolá-los, dificultando a construção de relações de confiança e reciprocidade.

Em síntese, esse elemento possibilita mapear as oportunidades de compartilhamento disponíveis, assim como verificar a adequação às necessidades de compartilhamento atuais para futuras intervenções. Além da falta de oportunidade de compartilhamento, os antecedentes consultados indicam uma série de possíveis barreiras ao compartilhamento de conhecimento, tanto para quem busca conhecimento, como para quem disponibiliza.

\subsection{Barreiras ao compartilhamento}

Os antecedentes consultados sugerem a existência de uma série de barreiras ao compartilhamento de conhecimento nas organizações, tanto para quem busca ou recebe conhecimento, quanto para quem disponibiliza (BURES, 2003; RIEGE, 2005; TONET; PAZ, 2006). Sob a ótica do compartilhamento de conhecimento como ação cooperativa, tais barreiras compõem parte dos custos envolvidos no ato de compartilhar conhecimento e devem ser mitigadas para que os indivíduos envolvidos optem mais facilmente por interações cooperativas com compartilhamento de conhecimento.

O que pode estar dificultando o compartilhamento? O que a organização pode fazer para mitigar essas barreiras? Há obstáculos para as fontes de conhecimento compartilharem? Há problemas para os destinatários de conhecimento buscarem e assimilarem os conhecimentos?

Ao buscar pelo conhecimento, o destinatário do processo pode sentir falta de tempo hábil para adquirir o conhecimento (ROSEN et al., 2007; SANTOS; SOARES; CARVALHO, 2012), assim como tempo hábil para identificar os colegas que possuem aquele conjunto de conhecimentos específicos ao qual necessita (LILLEOERE; HOLME HANSEN, 2011; RIEGE, 2005).

Perspectivas em Gestão \& Conhecimento, João Pessoa, v. 8, n. 1, p. 44-66, jan./abr. 2018. 
O tempo escasso para o compartilhamento é um dos principais gargalos para a efetivação desses processos de compartilhamento, desmotivando o destinatário que pode passar a perceber essas dificuldades como mais custos envolvidos no processo. Outra barreira que pode elevar esses custos percebidos é a falta de confiança na precisão e credibilidade das informações devido à fonte (RIEGE, 2005; ROSEN; FURST; BLACKBURN, 2007).

A confiança é um fator essencial para o compartilhamento de conhecimento (HSU; CHANG, 2014; LIN et al., 2009). Então a falta de confiança tanto entre quem busca e disponibiliza o conhecimento, quanto na utilidade e credibilidade do conhecimento compartilhado é uma barreira significativa a ser mitigada.

Nesse processo de busca pelo conhecimento, Tonet e Paz (2006) ainda indicam outras barreiras consideráveis como a dificuldade de comunicação e uso de linguagem e códigos comuns, pela fonte e pelo destinatário do conhecimento.

Ao compartilhar conhecimentos utilizando códigos e termos comuns aos destinatários do processo, estará possivelmente facilitando o entendimento e a absorção dos conhecimentos compartilhados.

O destinatário nesse caso pode perceber incompatibilidade na linguagem, nos códigos e na maneira de se comunicar da fonte de conhecimento, criando uma série de ruídos na comunicação que podem afetar a motivação e o comportamento de busca pelo conhecimento (HONG; SUH; KOO, 2011; SANTOS; SOARES; CARVALHO, 2012).

Essa barreira de comunicação pode se relacionar com as dificuldades de assimilação do destinatário pela falta de requisitos pessoais necessários ao uso do conhecimento a ser compartilhado, causando a resistência em adquirir o conhecimento, pelo fato de o destinatário ficar desconfortável em estar na posição de "aprendente" e não assimilar a nova aprendizagem (TONET; PAZ, 2006).

O tempo também é uma barreira restritiva para quem disponibiliza conhecimento (ROSEN; FURST; BLACKBURN, 2007; SANTOS; SOARES; CARVALHO, 2012). O ato de compartilhar conhecimento é entendido muitas vezes como trabalho adicional, pois geralmente não faz parte das atribuições formais do colaborador (BURES, 2003). Desta forma, quem disponibiliza conhecimento, seja face-a-face ou por intermédio de alguma tecnologia de comunicação, pode perceber o processo como um empecilho para sua atividade.

Outra barreira recorrentemente apontada na literatura é a retenção de conhecimento associada ao poder (BURES, 2003; LILLEOERE; HOLME HANSEN, 2011). O conhecimento é comumente associado a poder, pois no contexto de trabalho pode ser concebido com um diferencial para quem o detêm (LOUREIRO; CORRÊA, 2012). Um ativo que pode render benefícios se detido por poucas pessoas (fontes de conhecimento).

Além dessa associação com o poder, Riege (2005) aponta a possível existência de apreensão e medo de que o compartilhamento possa reduzir ou pôr em risco a segurança do emprego das pessoas, assim como o medo de não receber reconhecimento dos gerentes e colegas. Tonet e Paz (2006) acrescentam à associação de poder, o status relacionado ao detentor do conhecimento. Para os autores algumas fontes de conhecimento possuem resistência em repassar o conhecimento que detém por receio de perder o status perante $o$ grupo e por temer ser substituído pelo "aprendiz" (destinatário).

Essas barreiras associadas ao conhecimento como poder dentro de um ambiente organizacional, estão diretamente associadas à competitividade (SORDI; BINOTTO; RUVIARO, 2014). A competitividade interna e externa às unidades de negócio ou às áreas funcionais e entre filiais pode ser alta, dificultando o compartilhamento (RIEGE, 2005).

Sordi, Binotto e Ruviaro (2014) apresentam evidências de que o acirramento da competição entre esses grupos (departamentos, seções, equipes, unidades), podem diminuir o compartilhamento entre os grupos, e aumentar o compartilhamento interno. Ardichvili et al. (2006) relacionam a competitividade com a segurança do emprego. Onde um indivíduo fonte

Perspectivas em Gestão \& Conhecimento, João Pessoa, v. 8, n. 1, p. 44-66, jan./abr. 2018. 
de conhecimento, inserido num ambiente competitivo, não compartilha conhecimento visando a sustentação do cargo.

Outras barreiras relacionadas a não disponibilização de conhecimento são associadas a auto eficácia. Bures (2003) sugere que quando o indivíduo não está certo da utilidade de seu conhecimento ele não o compartilha para evitar constrangimentos. Os empregados mais jovens e menos experientes, por exemplo, podem se sentir inseguros quanto aos seus conhecimentos e se esses conhecimentos representam valor para os outros colaboradores.

Essa baixa consciência de realização do valor e dos benefícios do conhecimento possuído, podem funcionar como um impeditivo de compartilhamento de conhecimento por parte das fontes (RIEGE, 2005). Isso pode ser explicado, na visão de Lilleoere e Holme Hansen (2011), pelo medo de ser considerado tolo. No caso de a fonte ocupar um alto cargo hierárquico, esse medo pode ser o de demonstrar fraqueza (ARDICHVILI et al., 2006).

Assim como no caso dos destinatários, o relacionamento e a confiança podem funcionar como barreiras para o compartilhamento por parte das fontes de conhecimento. A falta de confiança nas pessoas pelo risco de elas empregarem mal esses conhecimentos ou tomarem os créditos para si, podem impossibilitar o compartilhamento de conhecimento (RIEGE, 2005).

Bures (2003) acrescenta o fato de alguns empregados visualizarem no compartilhamento de conhecimento uma maneira de perder as suas recompensas de trabalho ao disponibilizar seu conhecimento e experiência para alguém que pode ser recompensado com aumentos salariais. Essa visão causa incerteza e desconfiança, dificultando o ato de compartilhar (ALCARÁ et al., 2009).

Em síntese, esse elemento pode oferecer uma visão das possíveis barreiras existentes. E em quais barreiras a organização pode interferir no intuito de reduzi-las ou eliminá-las. Essas barreiras podem oferecer problemas às condições existentes para a cooperação entre os envolvidos no processo produtivo e, por consequência, dificultar os processos de compartilhamento de conhecimento entre eles.

\subsection{Condições para a existência de cooperação}

Sob a ótica do compartilhamento de conhecimento como ação cooperativa, é oferecendo condições para a existência de cooperação entre os indivíduos, que a organização irá possibilitar o compartilhamento de conhecimento entre eles.

O contexto estudado oferece as condições para a cooperação? Como a organização pode oferecer essas condições? Pela perspectiva adotada neste estudo, a existência dessas condições viabilizarão o compartilhamento de conhecimento entre os envolvidos.

Dessa maneira, a existência frequente de interações entre os atores e a valorização de interações futuras (AXELROD, 1997), sinais de reciprocidade (NOWAK, 2006), garantias, relações de dependência e arranjos institucionais (VILPOUX, 2014), são condições que favorecem a cooperação. E a organização pode criar mecanismos para oferecer essas condições visando facilitar a opção por interações cooperativas com compartilhamento de conhecimento entre os envolvidos.

Na perspectiva de Nowak (2005), as interações quando são frequentes e repetidas, permitem a existência de uma reputação entre os envolvidos e, no caso de uma reputação positiva, há a possibilidade de geração de confiança para futuras interações (AXELROD, 1997). A confiança entre esses indivíduos pode minimizar os riscos inerentes as interações e servir como garantias informais para futuras interações. Ou seja, havendo interações frequentes entre colaboradores, a chance do desenvolvimento de relações de confiança entre eles é maior. $\mathrm{O}$ que favorece o compartilhamento de conhecimentos por condicionar interações cooperativas, maximizando os benefícios percebidos em cooperar.

Perspectivas em Gestão \& Conhecimento, João Pessoa, v. 8, n. 1, p. 44-66, jan./abr. 2018. 
Essa pressão por cooperação (NOWAK, 2006), conjuntamente à reputação (AXELROD, 1997) oriunda das frequentes interações e a busca por reciprocidade, pode ser uma forma de seleção de grupo, ou seja, quem não se encaixar nesse contexto, segundo a perspectiva de Nowak (2006), cedo ou tarde sairá prejudicado e terá suas interações interrompidas. Desta forma, a opção por interações cooperativas se torna bem mais provável, elevando as condições de processos de compartilhamento de conhecimento acontecerem.

Pelo contrário, no caso de a relação que não oferecem interações frequentes, nem um conjunto de valores comuns, normas, códigos de comportamento e padrões que passam a ser socialmente aceitos, aprendidos e compartilhados (VILPOUX, 2014), a possibilidade de não haver cooperação e problemas de compartilhamento de conhecimento é substancialmente maior.

Esses conjuntos de códigos de conduta, segundo Ostrom (2000) e Vilpoux (2014), advêm de um arranjo informal entre os agentes, ordenando e definindo os limites de atuação e as formas de cooperação com base na confiança. Esses arranjos podem servir como garantias para a cooperação, e, consequentemente, favorecer o compartilhamento de conhecimento entre as partes envolvidas.

Dessa forma, é uma oportunidade de avaliar se o contexto analisado oferece as condições necessárias para a cooperação entre os envolvidos, possibilitando a reflexão desse contexto e o planejamento de intervenções. Esse elemento de análise fundamenta a perspectiva adotada neste estudo e busca reforçar a visão adotada do compartilhamento de conhecimento como ação cooperativa, por intermédio das possíveis sobreposições com os antecedentes consultados que compõem os demais elementos de análise tratados no modelo.

\subsection{Motivações para compartilhar}

Estudos anteriores sugerem que tanto as fontes, como os destinatários de conhecimento, necessitam estar motivados para que ocorra o compartilhamento. A motivação capta a disposição do indivíduo em compartilhar (SIEMSEN; ROTH; BALASUBRAMANIAN, 2008) e está relacionada a personalidade dos indivíduos (CABRERA; COLLINS; SALGADO, 2006), suas atitudes diante do compartilhamento de seus conhecimentos (CABRERA; CABRERA, 2005), às intenções de compartilhar ou não determinados conhecimentos (WITHERSPOON et al., 2013), aos seus comportamentos tanto como fontes ou destinatários (BOCK et al., 2005), aos seus interesses relativos ao compartilhamento (HEW; HARA, 2007) e aos sentimentos e emoções relacionados ao ato de compartilhar (VIDOTTI et al., 2016).

As pessoas estão motivadas a compartilhar? O que se pode alterar para aumentar essa motivação? $O$ que pode estar desmotivando as pessoas a compartilhar seus conhecimentos? A organização de alguma forma motiva o compartilhamento?

$\mathrm{Na}$ perspectiva adotada no modelo, a motivação trata da percepção do ato de compartilhar conhecimento pelos envolvidos (SIEMSEN; ROTH; BALASUBRAMANIAN, 2008) no que se refere às expectativas individuais tanto da fonte, como dos destinatários; e dos custos e benefícios percebidos por ambos em relação ao ato de compartilhar (CABRERA; CABRERA, 2005).

As motivações dos envolvidos nos processos de compartilhamento de conhecimento, permitem a avaliação do que pode estar motivando e do que pode estar desmotivando esses atos e comportamentos cooperativos.

\section{CONCLUSÃO}

Os resultados apresentados alcançaram o objetivo da pesquisa de propor um modelo analítico e empírico do compartilhamento de conhecimento nas organizações, baseando-se

Perspectivas em Gestão \& Conhecimento, João Pessoa, v. 8, n. 1, p. 44-66, jan./abr. 2018. 
em uma abordagem do compartilhamento de conhecimento como ação cooperativa. Esse modelo destaca que os principais focos para a análise desse processo são: as características do conhecimento a ser compartilhado, as características do ambiente organizacional, as oportunidades para compartilhar, as barreiras ao compartilhamento, as condições para a existência de cooperação e as motivações para compartilhar.

Os resultados ainda forneceram uma síntese das principais teorias utilizadas nos antecedentes literais consultados sobre o compartilhamento de conhecimento nas organizações e apresentaram a lógica dominante nessas mesmas publicações. E finalmente, os resultados sustentaram a premissa de que a gestão pode interferir positivamente nos processos de compartilhamento de conhecimento ao alterar as condições de cooperação existentes entre os indivíduos. Ou seja, que o compartilhamento de conhecimento necessita da cooperação entre os agentes para acontecer (SORDI; BINOTTO; RUVIARO, 2014).

Os gestores, analistas, pesquisadores e demais interessados podem utilizar o modelo proposto para identificar problemas relativos ao compartilhamento de conhecimento nas organizações e planejar possíveis soluções, medidas corretivas, ações ou políticas, fundamentadas na realidade de cada organização.

Por se tratar de uma extensa revisão crítica e narrativa dos antecedentes literais quanto ao compartilhamento de conhecimento nas organizações, o modelo emerge e está limitado ao conjunto de evidências empíricas e teóricas disponíveis na literatura consultada. 0 que não desvaloriza o arcabouço teórico resultante e também não impede a inclusão de dados empíricos adequados ao contexto de quem utilizará o modelo para analisar a ação cooperativa.

Desta maneira, sugerimos para estudos futuros a aplicação tanto do modelo analítico proposto, quanto da perspectiva adotada neste estudo, com o intuito de contribuir com evidências empíricas para o aprofundamento da temática. Estudos de multicasos, qualitativos e quantitativos, podem ser métodos a serem utilizados para esse fim.

Finalmente, é importante acrescentar que o uso do modelo de análise baseado em uma abordagem do compartilhamento de conhecimento como ação cooperativa, apresentado neste estudo, pode trazer resultados significativos em instituições que pretendem avaliar e analisar o seu próprio processo. Direcionando, com base nos resultados, ações para potencializar e fortalecer a ação cooperativa internamente e externamente.

\section{REFERÊNCIAS}

ABDUL-CADER, Khwaja M.; JOHAR, Gapar Md. A Comparison of Factors Influencing Knowledge Sharing through EKR among Sri Lankan and Singaporean Knowledge Workers. Management, v. 4, n. 6, 2015.

ALCARÁ, Adriana Rosecler et al. compartilhamento da informação e do conhecimento. Perspectivas em ciência da informação, v. 14, n. 1, p. 170-191, 2009.

AL SAIFI, Said Abdullah et al. The relationship between face to face social networks and knowledge sharing: an exploratory study of manufacturing firms. Journal of knowledge management, v. 20, n. 2, p. 308-326, 2016.

ALVES, Alessandra et al. Influências e barreiras ao compartilhamento da informação: uma perspectiva teórica. Ciência da Informação, Brasília, DF, v. 39, n. 2, p. 115-128, 2010.

Perspectivas em Gestão \& Conhecimento, João Pessoa, v. 8, n. 1, p. 44-66, jan./abr. 2018. 
ARDICHVILI, Alexandre et al. Cultural influences on knowledge sharing through online communities of practice. Journal of knowledge management, v. 10, n. 1, p. 94-107, 2006.

ARGOTE, Linda; MCEVILY, Bill; REAGANS, Ray. Managing knowledge in organizations: An integrative framework and review of emerging themes. Management science, v. 49, n. 4, p. 571-582, 2003.

AXELROD, Robert M. The complexity of cooperation: Agent-based models of competition and collaboration. Princeton University Press, 1997.

BARBOSA, José Geraldo Pereira et al. Compartilhamento de conhecimento: um estudo de caso em uma instituição financeira. Perspectivas em Gestão \& Conhecimento, v. 2, n. 1, p. 137-154, 2012.

BARTOL, Kathryn M.; SRIVASTAVA, Abhishek. Encouraging knowledge sharing: the role of organizational reward systems. Journal of Leadership \& Organizational Studies, v. 9, n. 1, p. 64-76, 2002.

BLOICE, Lyndsay; BURNETT, Simon. Barriers to knowledge sharing in third sector social care: a case study. Journal of Knowledge Management, v. 20, n. 1, p. 125-145, 2016.

BOCK, Gee-Woo et al. Behavioral intention formation in knowledge sharing: Examining the roles of extrinsic motivators, social-psychological forces, and organizational climate. MIS quarterly, p. 87-111, 2005.

BRACHOS, Dimitris et al. Knowledge effectiveness, social context and innovation. Journal of knowledge management, v. 11, n. 5, p. 31-44, 2007.

BUREŠ, Vladimír. Cultural barriers in knowledge sharing. E+ M Ekonomics and Management, Liberec, v. 6, p. 57-62, 2003.

CABRERA, Angel; COLLINS, William C.; SALGADO, Jesús F. Determinants of individual engagement in knowledge sharing. The International Journal of Human Resource Management, v. 17, n. 2, p. 245-264, 2006.

CABRERA, Elizabeth F.; CABRERA, Angel. Fostering knowledge sharing through people management practices. The International Journal of Human Resource Management, v. 16, $n$. 5, p. 720-735, 2005.

CASIMIR, Gian; LEE, Karen; LOON, Mark. Knowledge sharing: influences of trust, commitment and cost. Journal of Knowledge Management, v. 16, n. 5, p. 740-753, 2012.

CAVALIERE, Vincenzo; LOMBARDI, Sara; GIUSTINIANO, Luca. Knowledge sharing in knowledgeintensive manufacturing firms. An empirical study of its enablers. Journal of Knowledge Management, v. 19, n. 6, p. 1124-1145, 2015.

CHEN, Cheng-Wu; CHANG, Min-Li; TSENG, Chun-Pin. Human factors of knowledge-sharing intention among taiwanese enterprises: A model of hypotheses. Human Factors and Ergonomics in Manufacturing \& Service Industries, v. 22, n. 4, p. 362-371, 2012.

Perspectivas em Gestão \& Conhecimento, João Pessoa, v. 8, n. 1, p. 44-66, jan./abr. 2018. 
CHIU, Chao-Min; HSU, Meng-Hsiang; WANG, Eric TG. Understanding knowledge sharing in virtual communities: An integration of social capital and social cognitive theories. Decision support systems, v. 42, n. 3, p. 1872-1888, 2006.

CORREIA, Rony Rodrigues. Associações entre princípios sociotécnicos e compartilhamento de conhecimento: Estudo de caso em projetos de sistemas de informação. Perspectivas em Gestão \& Conhecimento, v. 3, n. 1, p. 175-191, 2013.

CUMMINGS, Jonathon N. Work groups, structural diversity, and knowledge sharing in a global organization. Management science, v. 50, n. 3, p. 352-364, 2004.

DAVENPORT, Thomas H.; PRUSAK, Laurence. Working knowledge: How organizations manage what they know. Harvard Business Press, 2000.

DOROW , Patrícia Fernanda; CALLE, Guillermo Antonio Dávila; RADOS, Gregório Jean Varvakis. O Ciclo de conhecimento como gerador de valor: Uma proposta integradora. Revista Espacios, v. 36, n. 12, p.126, 2015.

DURKHEIM, Émile. As regras do método sociológico. São Paulo: Martin Claret, 2001.

GRASSI, Robson Antonio. Capacitações dinâmicas, coordenação e cooperação interfirmas: as visões Freeman-Lundvall e Teece-Pisano. Estudos Econômicos (São Paulo), v. 36, n. 3, p. 611635, 2006.

HALL, Hazel. Input-friendliness: motivating knowledge sharing across intranets. Journal of information science, v. 27, n. 3, p. 139-146, 2001.

$\mathrm{HE}$, Wei; WEl, Kwok-Kee. What drives continued knowledge sharing? An investigation of knowledge-contribution and-seeking beliefs. Decision Support Systems, v. 46, n. 4, p. 826-838, 2009.

HEW, Khe Foon; HARA, Noriko. Empirical study of motivators and barriers of teacher online knowledge sharing. Educational Technology Research and Development, v. 55, n. 6, p. 573595, 2007.

HENDRIKS, Paul. Why share knowledge? The influence of ICT on the motivation for knowledge sharing. Knowledge and process management, v. 6, n. 2, p. 91-100, 1999.

HONG, Daegeun; SUH, Euiho; KOO, Choonghyo. Developing strategies for overcoming barriers to knowledge sharing based on conversational knowledge management: A case study of a financial company. Expert systems with Applications, v. 38, n. 12, p. 14417-14427, 2011.

HSU, Meng-Hsiang; CHANG, Chun-Ming. Examining interpersonal trust as a facilitator and uncertainty as an inhibitor of intra-organisational knowledge sharing. Information Systems Journal, v. 24, n. 2, p. 119-142, 2014.

HSU, Chin-Lung; LIN, Judy Chuan-Chuan. Acceptance of blog usage: The roles of technology acceptance, social influence and knowledge sharing motivation. Information \& management, v. 45, n. 1, p. 65-74, 2008.

Perspectivas em Gestão \& Conhecimento, João Pessoa, v. 8, n. 1, p. 44-66, jan./abr. 2018. 
IPE, Minu. Knowledge sharing in organizations: A conceptual framework. Human resource development review, v. 2, n. 4, p. 337-359, 2003.

KIM, Soonhee; LEE, Hyangsoo. The impact of organizational context and information technology on employee knowledge-sharing capabilities. Public Administration Review, v. 66, n. 3, p. 370-385, 2006.

KIM, Yong-Mi; NEWBY-BENNETT, Donna; SONG, Hee-Joon. Knowledge sharing and institutionalism in the healthcare industry. Journal of Knowledge Management, v. 16, n. 3, p. 480-494, 2012.

KYOON YOO, Dong; VONDEREMBSE, Mark A.; RAGU-NATHAN, T. S. Knowledge quality: antecedents and consequence in project teams. Journal of Knowledge Management, v. 15, n. 2, p. 329-343, 2011.

LAWSON, Benn et al. Knowledge sharing in interorganizational product development teams: the effect of formal and informal socialization mechanisms*. Journal of Product Innovation Management, v. 26, n. 2, p. 156-172, 2009.

LIAO, Li-Fen. Knowledge-sharing in R\&D departments: A social power and social exchange theory perspective. The International Journal of Human Resource Management, v. 19, n. 10, p. 1881-1895, 2008.

LILLEOERE, Anne-Mette; HOLME HANSEN, Ebba. Knowledge-sharing enablers and barriers in pharmaceutical research and development. Journal of Knowledge Management, v. 15, n. 1, p. 53-70, 2011.

LIN, Hsiu-Fen. Effects of extrinsic and intrinsic motivation on employee knowledge sharing intentions. Journal of information science, 2007.

LIN, Hsiu-Fen. Knowledge sharing and firm innovation capability: an empirical study. International Journal of manpower, v. 28, n. 3/4, p. 315-332, 2007.

LIN, Ming-Ji James; HUNG, Shiu-Wan; CHEN, Chih-Jou. Fostering the determinants of knowledge sharing in professional virtual communities. Computers in Human Behavior, v. 25, n. 4, p. 929-939, 2009.

LIN, Tung-Ching; WU, Sheng; LU, Chun-Tai. Exploring the affect factors of knowledge sharing behavior: The relations model theory perspective. Expert Systems with Applications, v. 39, n. 1, p. 751-764, 2012.

LOUREIRO, Rogério Salles; CORRÊA, Dalila Alves. Compartilhamento de conhecimento com foco no processo de socialização e sustentabilidade em projetos de inovação: Um estudo de caso no centro de tecnologia canavieira. Caderno Profissional de Administração da UNIMEP, v. 2, n. 2, 2012.

MA, Zhenzhong et al. What matters for knowledge sharing in collectivistic cultures? Empirical evidence from China. Journal of Knowledge Management, v. 18, n. 5, p. 1004-1019, 2014.

Perspectivas em Gestão \& Conhecimento, João Pessoa, v. 8, n. 1, p. 44-66, jan./abr. 2018. 
NONAKA, Ikujiro; VON KROGH, Georg. Perspective-tacit knowledge and knowledge conversion: Controversy and advancement in organizational knowledge creation theory. Organization science, v. 20, n. 3, p. 635-652, 2009.

NOWAK, Martin A. Five rules for the evolution of cooperation. Science, v. 314, n. 5805, p. 1560-1563, 2006

OSTROM, Elinor. Collective action and the evolution of social norms. The Journal of Economic Perspectives, p. 137-158, 2000.

POPADIUK, Silvio; CHOO, Chun Wei. Innovation and knowledge creation: How are these concepts related?. International journal of information management, v. 26, n. 4, p. 302-312, 2006.

PULAKOS, Elaine D.; DORSEY, David W.; BORMAN, Walter C. Hiring for knowledge-based competition. Managing knowledge for sustained competitive advantage: Designing strategies for effective human resource management, p. 155-176, 2003.

RICHTER, Rudolf. New economic sociology and new institutional economics. In: Essays on New Institutional Economics. Springer International Publishing, 2015. p. 51-75.

RIEGE, Andreas. Three-dozen knowledge-sharing barriers managers must consider. Journal of knowledge management, v. 9, n. 3, p. 18-35, 2005.

RIVERA-VAZQUEZ, Juan C.; ORTIZ-FOURNIER, Lillian V.; ROGELIO FLORES, Felix. Overcoming cultural barriers for innovation and knowledge sharing. Journal of Knowledge Management, $v$. 13, n. 5, p. 257-270, 2009.

ROSEN, Benson; FURST, Stacie; BLACKBURN, Richard. Overcoming barriers to knowledge sharing in virtual teams. Organizational Dynamics, v. 36, n. 3, p. 259-273, 2007.

ROTHER, Edna Terezinha. Revisão sistemática $X$ revisão narrativa. Acta paulista de enfermagem, v. 20, n. 2, p. v-vi, 2007.

RUTTEN, Werner et al. The impact of (low) trust on knowledge sharing. Journal of knowledge management, v. 20, n. 2, p. 199-214, 2016.

RYU, Seewon; HO, Seung Hee; HAN, Ingoo. Knowledge sharing behavior of physicians in hospitals. Expert Systems with applications, v. 25, n. 1, p. 113-122, 2003.

SALIM, M. et al. Antecedents of Knowledge Sharing Attitude and Intentions. European Journal of Scientific Research, v. 56, n. 1, p. 44-50, 2011.

SANTOS, Vitor Ricardo; SOARES, António Lucas; CARVALHO, João Álvaro. Knowledge sharing barriers in complex research and development projects: An exploratory study on the perceptions of project managers.Knowledge and Process Management, v. 19, n. 1, p. 27-38, 2012. 
SENTHIL VELMURUGAN, Manivannan; KOGILAH, Narayanasamy; DEVINAGA, Rasiah. Knowledge Sharing in Virtual Teams in Malaysia: its benefits and barriers. Journal of Information \& Knowledge Management, v. 9, n. 02, p. 145-159, 2010.

SIEMSEN, Enno; ROTH, Aleda V.; BALASUBRAMANIAN, Sridhar. How motivation, opportunity, and ability drive knowledge sharing: The constraining-factor model. Journal of Operations Management, v. 26, n. 3, p. 426-445, 2008.

SORDI, V. F.; NAKAYAMA, M. K.; de ALMEIDA CUNHA, C. J. C.; BINOTTO, E. Fatores determinantes ao compartilhamento de conhecimento nas organizações: a perspectiva bidirecional. Revista Eletrônica de Estratégia \& Negócios, v. 10, n. 2, p. 225-246, 2017.

SORDI, Victor Fraile; BINOTTO, Erlaine; RUVIARO, Clandio Favarini. A cooperação e o compartilhamento de conhecimentos em uma cooperativa de crédito. Perspectivas em Gestão \& Conhecimento, v. 4, n. 1, p. 119-134, 2014.

STENIUS, Minna et al. Why share expertise? A closer look at the quality of motivation to share or withhold knowledge. Journal of Knowledge Management, v. 20, n. 2, p. 181-198, 2016.

SZULANSKI, Gabriel. The process of knowledge transfer: A diachronic analysis of stickiness. Organizational behavior and human decision processes, v. 82, n. 1, p. 9-27, 2000.

TANGARAJA, Gangeswari et al. Knowledge sharing is knowledge transfer: a misconception in the literature. Journal of Knowledge Management, v. 20, n. 4, p. 653-670, 2016.

TONET, Helena Correa; PAZ, Maria das Graças Torres da. Um modelo para o compartilhamento de conhecimento no trabalho. Revista de Administração Contemporânea, v. 10, n. 2, p. 75-94, 2006.

VAN DEN HOOFF, Bart; DE LEEUW VAN WEENEN, Femke. Committed to share: commitment and $\mathrm{CMC}$ use as antecedents of knowledge sharing. Knowledge and Process Management, $v$. 11, n. 1, p. 13-24, 2004.

VIDOTTI, Amanda F. et al. Emoções Positivas no Compartilhamento do Conhecimento Organizacional. In: VI Congreso Internacional de Conocimiento e Innovación 31 octubre - 01 noviembre, 2016, Bogotá. Proceeding of the 6 International Congress of Knowledge and Inovation (ciKi) (31 of October and 01 of November of 2016, Bogotá, Colombia). Florianópolis: EGC/UFSC, 2016".

VILPOUX, Olivier F. Agrarian reform and cooperation between settlers in the Midwest of Brazil: An institutional approach. Land Use Policy, v. 39, p. 65-77, 2014.

VON BERTALANFFY, Ludwig. Teoria geral dos sistemas. Vozes, 2008.

WANG, Sheng; NOE, Raymond A. Knowledge sharing: A review and directions for future research. Human Resource Management Review, v. 20, n. 2, p. 115-131, 2010.

WEBER, Max. Economy and society: An outline of interpretive sociology. Univ of California Press, 1978.

Perspectivas em Gestão \& Conhecimento, João Pessoa, v. 8, n. 1, p. 44-66, jan./abr. 2018. 
WILLIAMSON, Oliver. The mechanisms of governance. Oxford University Press, 1996.

WITHERSPOON, Candace L. et al. Antecedents of organizational knowledge sharing: a metaanalysis and critique. Journal of Knowledge Management, v. 17, n. 2, p. 250-277, 2013.

YUSOF, Zawiyah M. et al. Knowledge sharing in the public sector in Malaysia a proposed holistic model. Information Development, v. 28, n. 1, p. 43-54, 2012.

ZÁRRAGA, Celia; BONACHE, Jaime. Assessing the team environment for knowledge sharing: an empirical analysis. International Journal of Human Resource Management, v. 14, n. 7, p. 1227-1245, 2003.

ZHANG, Jing; DAWES, Sharon S.; SARKIS, Joseph. Exploring stakeholders' expectations of the benefits and barriers of e-government knowledge sharing.Journal of Enterprise Information Management, v. 18, n. 5, p. 548-567, 2005.

Artigo recebido em 17/01/2017 e aceito para publicação em 19/02/2018 